\title{
Simplified Modelling of Legs Dynamics on Quadruped Robots' Force Control Approach
}

\author{
José L. Silvino ${ }^{1}$, Peterson Resende ${ }^{1}$, \\ Luiz S. Martins-Filho ${ }^{2}$ and Tarcísio A. Pizziolo ${ }^{3}$ \\ ${ }^{1}$ Univ. Federal de Minas Gerais, ${ }^{2}$ Univ. Federal de Ouro Preto, ${ }^{3}$ Univ. Federal de Viçosa \\ Brazil
}

\section{Introduction}

The development of the service and intervention robotics has stimulated remarkable projects of mobile robots well adapted to different kinds of environment, including structured and non-structured terrains. On this context, several control system architectures have been proposed looking for the improvement of the robot autonomy, and of the tasks planning capabilities, as well reactive characteristics to deal with unexpected events (Medeiros et al., 1996; Martins-Filho \& Prajoux, 2000). The proposed solutions to the locomotion on hazardous and strongly irregular soils include adapted wheels robots, rovers, robots equipped with caterpillar systems, and walking robots. Some of this robot designs are inspired on successful locomotion systems of mammals and insects.

The legged robots have obtained promising results when dealing with terrains presenting high degrees of difficulty. This is quite curious to notice that ideas concerning this robotic

$\varepsilon$ locomotion system have been present since the first idealistic dreams of the robotics history,

0 and nowadays this approach has gained the interest and attention of numerous researchers

$\stackrel{\oplus}{.}$ and laboratories.

Let's mention some of the relevant works involving legged robots: Hirose et al. (1989) proposes an architecture for control and supervision of walking; Klein and Q Kittivatcharapong (1990) study optimal distribution of the feet-soil contact forces; Vukobratovic et al. (1990) work on the robot dynamics modelling and control; Pack and Kang (1995) discuss the strategies of walk control concerning the gaits; Perrin et al. (1997) propose a detailed platform and legs mechanisms modelling for the dynamics simulation; \& Tanie (2001) discusses the new perspectives and trends for the walking machines; Schneider 政 and Schmucker (2001) work on force control of the complete robot mechanical system.

1.1 The Context of this Study

\& A walking robot can be described as a multibody chained dynamical system, consisting of a \& platform (the robot body) and a number of leg mechanisms that are similar to manipulator robotic arms. Considering the locomotion control of robots with significant masses, the main О. approaches are based on force control, this means that the leg active joints actuators produce torques and/or forces resulting on contact forces in the feet-soil contacts. For instance, this Source: Climbing \& Walking Robots, Towards New Applications, Book edited by Houxiang Zhang, ISBN 978-3-902613-16-5, pp.546, October 2007, Itech Education and Publishing, Vienna, Austria 
principle of locomotion control can be seen in (Hirose et al., 1989; Martins-Filho \& Prajoux, 2000; Schneider \& Schmucker 2001). As a consequence, the system produces angular and linear accelerations on the chained mechanism components, and the robot moves to execute the required locomotion task.

Evidently, on this control approach, for the control of the walking robot position and velocity, the system should have the robot dynamics model to allow the efforts determination that must be produced by the joint actuators (Cunha et al., 1999). The dynamics model provides the relation between the robot state variables (acceleration, velocity, position) and the active joint torques/forces, taking into account the robot design, geometry, masses and inertias and other physical characteristics.

The main purpose of this work is the careful analysis of the effects of an eventual simplification on the dynamical equations of a small quadruped robot. The simplification effects are verified through the comparison between results of numerical simulations of the complete dynamical model, and of the simplified model, where the $C(q, d q / d t)$ and $G(q)$ are negligible.

The Fig. 1 shows the general aspects of the design of the considered quadruped robot. As can be seen, this robot is composed by a square platform, called the robot body, supported by four identical leg mechanisms. The mechanical design of each 3-joints leg is depicted on the Fig. 2.

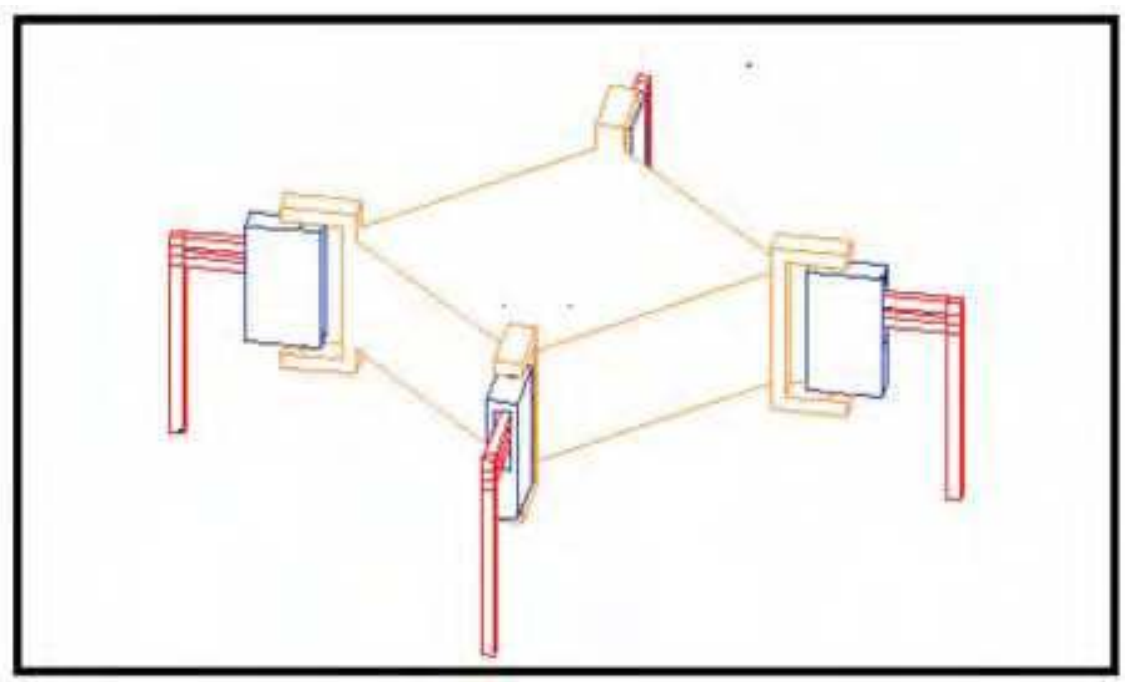

Fig. 1. The design of the studied quadruped robot

The Sect. 2 presents the dynamical model of the leg mechanisms, discusses the computation of important matrices appearing in this model, and analyses the workspace of the proposed leg design. The analysis of details of the dynamical model, that can be simplified considering the physical characteristics of the studied walking robot, is shown in Sect. 3 . The following section (Sect. 4) describes the numerical simulations, and presents the obtained results and its analysis. The Sect. 5 closes the chapter with the work conclusions and comments about the future works on this research subject. 


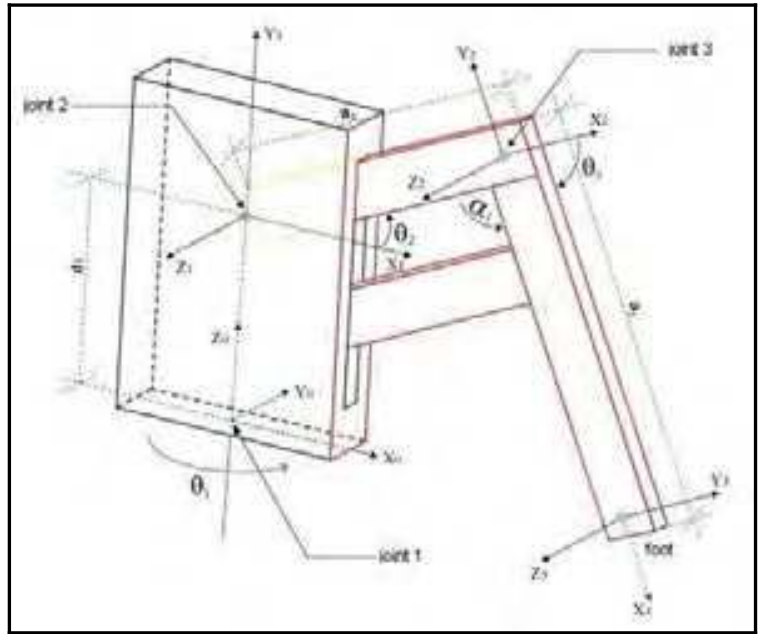

Fig. 2. The proposed prototype design of the leg mechanism

\section{Dynamical Modelling of the Leg Mechanism}

The leg mechanism model is based on the actuators dynamics, composed by servomotors and reductions gears, as well the friction effects on this active joints (Coulomb and viscous friction). Moreover, all the robot's links are taken as been completely rigid, a usual model assumption.

Let $q_{i}=\theta_{i}$ the $i$-th rotational joint angle, the complete state configuration of each leg is defined by a vector of generalized coordinates as follows:

$$
q=\left[\begin{array}{lll}
\theta_{1} & \theta_{2} & \theta_{3}
\end{array}\right]^{T}
$$

Considering the kinematics energy, $E_{c}$, and the potential energy, $E_{p}$, the conservative Lagrangian for the system is given by:

$$
L(q, \dot{q})=E_{c}-E_{p}
$$

And the equations of motion of this dynamical system are described as follows:

$$
\frac{d}{d t}\left(\frac{\partial L}{\partial \dot{q}_{i}}\right)-\frac{\partial L}{\partial q_{i}}=Q_{i}
$$

where $L(q, d q / d t)=E_{c}-E_{p}$, and $Q_{i}$ is the vector of generalized force corresponding to the generalized coordinate $q_{i}$. The kinematics energy of each leg mechanism is obtained by the summation of the leg links energies, $K_{c i}$. 
Considering the linear velocity of each link's centre-of-mass, the vector $d p / d t$, and the angular velocity $\omega_{i}$, the resulting equation is:

$$
\begin{aligned}
\dot{p}_{i} & =J_{L_{1}}^{(i)} \cdot \dot{q}_{1}+J_{L_{2}}^{(i)} \cdot \dot{q}_{2}+J_{L_{3}}^{(i)} \cdot \dot{q}_{3} \\
w_{i} & =J_{A_{1}}^{(i)} \cdot \dot{q}_{1}+J_{A_{2}}^{(i)} \cdot \dot{q}_{2}+J_{A_{3}}^{(i)} \cdot \dot{q}_{3}
\end{aligned}
$$

$J_{L_{1}}^{(i)}, J_{L_{2}}^{(i)}, J_{L_{3}}^{(i)}$ are the $i$-th row vectors of the matrix $J$ (dimension $3 \times 3$ ) for the linear velocities of the links 1, 2 and 3, and $J_{A_{1}}^{(i)}, J_{A_{2}}^{(i)}, J_{A_{3}}^{(i)}$ are the $i$-th row vectors of the matrix $J$ for the angular velocities of the links 1,2 and 3. The kinematics energy of each link results of the translational and rotational terms:

$$
K_{i}=\frac{1}{2} \dot{p}_{i}^{T} \cdot m_{i} \cdot \dot{p}_{i}+\frac{1}{2} \omega_{i}^{T} \cdot I_{i} \cdot \omega_{i}
$$

where $m_{i}$ and $I_{i}$ are the mass and the inertia tensor expressed in the base coordinates system, respectively. Applying the results of Eq. (4) in the Eq. (5), the expression for the total kinetics energy of each three degrees-of-freedom leg.

$$
K=\frac{1}{2} \sum_{i=1}^{3}\left(\dot{q}^{T} \cdot J_{L}^{(i))^{T}} \cdot m_{i} \cdot J_{L}^{(i)} \cdot \dot{q}+\dot{q}^{T} \cdot J_{A}^{(i)^{T}} \cdot I_{i} \cdot J_{A}^{(i)} \cdot \dot{q}\right)
$$

The term $H(q)$ can be defined as a symmetric square matrix based on the each link's tensor of inertia. Consequently, is possible to obtain:

$$
H(q)=\sum_{i=1}^{3}\left(J_{L}^{(i)^{T}} \cdot m_{i} \cdot J_{L}^{(i)}+J_{A}^{(i)^{T}} \cdot I_{i} \cdot J_{A}^{(i)}\right)
$$

The matrix $H(q)$ represents the mass characteristics of the leg mechanism. This matrix is called matrix of inertia tensor. The matrix elements $H_{i i}(q)$ are related to the effective inertias, and the $H_{i j}(q)$, with $i \neq j$, are related to the coupling inertia. Using these properties, the Eq. (5) can be re-written in a compact form:

$$
K=\frac{1}{2} \dot{q}^{T} H(q) \cdot \dot{q}
$$

The potential energy $E_{p}$, considering a leg mechanism composed by rigid links, is function exclusively of the gravity. The vector $g$ represents the gravitational acceleration. The overall potential energy of each leg is given by:

$$
E_{p}=\sum_{i=1}^{3} m_{i} \cdot g^{T} \cdot r_{i}
$$


where $r_{i}$ is the position of the centre-of-mass of each link, described in the base coordinates system.

The Lagrangian formulation provides the motion equations of the robotic leg mechanism system, using the kinematics and potential energies, the forces and torques actuating on the leg (excluding the gravitational and inertial forces, i.e. the generalized forces). This formulation results in the following equation:

$$
\frac{d}{d t}\left(\frac{\partial L}{\partial \dot{q}_{i}}\right)-\frac{\partial L}{\partial q_{i}}=\frac{d}{d t}\left(\frac{\partial E_{c}}{\partial \dot{q}_{i}}-\frac{\partial E_{p}}{\partial \dot{q}_{i}}\right)-\left(\frac{\partial E_{c}}{\partial q_{i}}-\frac{\partial E_{p}}{\partial q_{i}}\right)=Q_{i}
$$

Considering that the derivative of $E_{c}$ is obtained as follows:

$$
\frac{d}{d t}\left(\frac{\partial E_{c}}{\partial \dot{q}_{i}}\right)=\frac{d}{d t}\left(\sum_{j=1}^{3} H_{i j} \cdot \dot{q}_{j}\right)=\sum_{j=1}^{3} H_{i j} . . \ddot{q}_{j}+\sum_{j=1}^{3} \frac{d H_{i j}}{d t} \cdot \dot{q}_{j}
$$

the time derivative of $H_{i j}$ is given by:

$$
\begin{aligned}
& \frac{d H_{i j}}{d t}=\sum_{j=1}^{3} \frac{\partial H_{i j}}{\partial q_{k}} \cdot \frac{d q_{k}}{d t}=\sum_{j=1}^{3} \frac{\partial H_{i j}}{\partial q_{k}} \cdot \dot{q}_{k} \\
& \frac{d}{d t}\left(\frac{\partial E_{P}}{\partial q_{i}}\right)=0 \\
& \frac{\partial E_{c}}{\partial \dot{q}_{i}}=\frac{\partial}{\partial q_{i}}\left(\frac{1}{2} \sum_{j=1}^{3} \sum_{k=1}^{3} H_{j k} \cdot \dot{q}_{j} \cdot \dot{q}_{k}\right)=-\frac{1}{2} \sum_{j=1}^{3} \sum_{i=1}^{3} \frac{\partial H_{j k}}{\partial q_{i}} \cdot \dot{q}_{j} \cdot \dot{q}_{i} \\
& \frac{\partial E_{P}}{\partial q_{i}}=\sum_{j=1}^{3} m_{j} \cdot g^{T} \cdot \frac{\partial r_{j}}{\partial q_{i}}
\end{aligned}
$$

For the Eq. (14), the partial derivative of $r_{i j}$ with respect to $q_{i}$ is equal to the $j$-th component of the $i$-th column of the Jacobian matrix $J_{l}$ (linear velocities). The equation becomes:

$$
\frac{d H_{i j}}{d t}=\sum_{j=1}^{3} \frac{\partial H_{i j}}{\partial q_{k}} \cdot \frac{d q_{k}}{d t}=\sum_{j=1}^{3} \frac{\partial H_{i j}}{\partial q_{k}} \cdot \dot{q}_{k}
$$

This term is called gravitational term, and it is represented by $G_{i}$ : 


$$
G_{i}=\sum_{j=1}^{3}\left(m_{j} \cdot g^{T} \cdot J_{L i}^{(i)}\right)
$$

Considering the original equation of the Lagragian formulation (Eq. (9)), and taking into account the last developments, the resulting equation is given by:

$$
\sum_{j=1}^{3} H_{i j} \cdot \ddot{q}_{j}+\sum_{j=1}^{3} \sum_{k=1}^{3} h_{i j k} \cdot \dot{q}_{j} \cdot \dot{q}_{k}+G_{i}=Q_{i}
$$

where $h_{i j k}=\partial H_{i j} / \partial q_{k}$

The Eq. (17) can be rewritten under a compact form as follows:

$$
D(q) \cdot \ddot{q}+C(q, \dot{q}) \cdot \dot{q}+G(q)=u+J(q)^{T} \cdot F_{e}
$$

$D(q)$ is a matrix $3 \times 3$ that represents the inertial torques, including the torques resulting of link interactions; $C(q, d q / d t)$ is a matrix $3 \times 3$ that represents the centrifugal and Coriolis forces; $U$ is a vector $3 \times 1$ of control torques (to be defined by the robot control function), $J(q)$ is the Jacobian matrix, also with dimension $3 \times 3$, and $F$ is the vector $3 \times 1$ of generalized forces/torques produced by the environment of the work space (this vector is expressed in the base coordinate system) (Asada, 1986; Cunha et al. 1999).

\subsection{Computation of the Matrices $D(q), C(q, d q / d t)$ and $G(q)$}

The locomotion system of the considered quadruped robot controls independently each one of the leg mechanisms and their active joints. As a consequence, the overall robot control can be divided into the leg subsystems and integrated by the resulting efforts on the hips, finally closing the chained system. Based on this principle, the modelling of robot dynamics will consider the leg mechanisms initially independently. For the derivation of this leg model, it's necessary to obtain the matrices $D(q), C(q, d q / d t)$, and $G(q)$. Theses matrices expressions are determined by the equations of the direct kinematics for the proposed robot design. Adopting the Denavit-Hartenberg convention for manipulator robots (Spong \& Vidyasagar, 1989), the direct kinematics provides the vector $x$ of the leg-end position, $x=\left[\begin{array}{lll}x_{P} & y_{P} & z_{P}\end{array}\right]^{T}$. The expression of this vector is:

$$
x=\left[\begin{array}{l}
x_{P} \\
y_{P} \\
z_{P}
\end{array}\right]=\left[\begin{array}{l}
a_{3} c_{1} c_{2} c_{3}-a_{3} c_{1} s_{2} s_{3}+a_{2} c_{1} c_{2} \\
a_{3} s_{1} c_{2} c_{3}-a_{3} s_{1} s_{2} s_{3}+a_{2} s_{1} c_{2} \\
a_{3} s_{2} c_{3}+a_{3} c_{2} s_{3}+a_{2} s_{2}+d_{1}
\end{array}\right]
$$

where a compact notation was adopted to simplify the equation: $c_{i}=\cos \left(\theta_{I}\right), \quad c_{i j}=\cos \left(\theta_{i}+\theta_{j}\right)$, $s_{i}=\sin \left(\theta_{1}\right), s_{i j}=\sin \left(\theta_{i}+\theta_{j}\right)$. The Jacobian matrix $J(q)$ is determined as follows: 


$$
J=\left[\begin{array}{ccc}
-a_{2} s_{1} c_{2}-a_{3} s_{1} c_{23} & -a_{2} c_{1} s_{1}-a_{3} c_{1} s_{23} & -a_{3} c_{1} s_{23} \\
a_{2} c_{1} c_{2}+a_{3} c_{1} c_{23} & -a_{2} s_{1} s_{2}-a_{3} s_{1} s_{23} & -a_{3} s_{1} s_{23} \\
0 & a_{2} c_{2}+a_{3} c_{23} & a_{3} s_{2} c_{23}
\end{array}\right]
$$

The linear and angular velocities of the leg links, with respect to their centres-of-mass, are given by:

$$
\begin{gathered}
\dot{p}_{1}=\left[\begin{array}{lll}
0 & 0 & 0 \\
0 & 0 & 0 \\
0 & 0 & 0
\end{array}\right] \cdot \dot{q} \\
p_{2}=\left[\begin{array}{ccc}
-r_{2} s_{1} c_{2} & -r_{2} c_{1} s_{1} & 0 \\
r_{1} c_{1} c_{2} & -r_{2} s_{1} s_{2} & 0 \\
0 & r_{2} c_{2} & 0
\end{array}\right] \cdot \dot{q} \\
p_{3}=\left[\begin{array}{ccc}
-a_{2} s_{1} c_{2}-r_{3} s_{1} c_{23} & -a_{2} c_{1} s_{1}-r_{3} c_{1} s_{23} & -r_{3} c_{1} s_{23} \\
a_{2} c_{1} c_{2}+r_{3} c_{1} c_{23} & -a_{2} s_{1} s_{2}-r_{3} s_{1} s_{23} & -r_{3} s_{1} s_{23} \\
0 & a_{2} c_{2}+r_{3} c_{23} & r_{3} s_{2} c_{23}
\end{array}\right] \cdot \dot{q} \\
w_{1}=\left[\begin{array}{lll}
1 & 0 & 0
\end{array}\right] \cdot \dot{q} \\
w_{2}=\left[\begin{array}{lll}
1 & 1 & 0
\end{array}\right] \cdot \dot{q} \\
w_{3}=\left[\begin{array}{lll}
1 & 1 & 1
\end{array}\right] \cdot \dot{q}
\end{gathered}
$$

The matrix $D(q)$ can be now determined. Its expression is given by:

$$
D(q)=\left(m_{1} \cdot p_{1}^{T} \cdot p_{1}+I_{1} \cdot w_{1}^{T} \cdot w_{1}\right)+\left(m_{2} \cdot p_{2}^{T} \cdot p_{2}+I_{2} \cdot w_{2}^{T} \cdot w_{2}\right)+\left(m_{3} \cdot p_{3}^{T} \cdot p_{3}+I_{3} \cdot w_{3}^{T} \cdot w_{3}\right)
$$

where $m_{1}, m_{2}$ and $m_{3}$ are mass value of the links, and $I_{1}, I_{2}$ and $I_{3}$ are the moments of inertia with respect to the centre-of-mass of each leg link.

The matrix $C(q, d q / d t)$ is composed by the elements $h_{i j k}$ that are multiplied by the vector $d q / d t$. The elements $h_{i j k}$ are determined using the matrix $D(q)$ thought the relation $h_{i j k}=\left(\partial \mathrm{D}_{i j} / \partial q_{k}\right)$. Consequently, is possible to obtain: 


$$
C(q, q)=\left[\begin{array}{ccc}
\sum_{k=1}^{3} h_{11 k} \cdot q_{k} & \sum_{k=1}^{3} h_{12 k} \cdot q_{k} & \sum_{k=1}^{3} h_{13 k} \cdot q_{k} \\
\sum_{k=1}^{3} h_{21 k} \cdot q_{k} & \sum_{k=1}^{3} h_{22 k} \cdot q_{k} & \sum_{k=1}^{3} h_{231} \cdot q_{k} \\
\sum_{k=1}^{3} h_{31 k} \cdot q_{k} & \sum_{k=1}^{3} h_{32 k} \cdot q_{k} & \sum_{k=1}^{3} h_{33 k} \cdot q_{k}
\end{array}\right]
$$

The matrix $G(q)$ is given by the expression of the gravitational contributions $G_{i}=m_{j} g T J_{L_{i}}^{(i)}$.

Taking the equations of the system dynamics, the robot system states can be obtained directly by the expression of the joints acceleration $d^{2} q / d t^{2}$. This expression is given by:

$$
\ddot{q}=D(q)^{-1} \cdot[-C(q, q) \cdot \dot{q}]+D(q)^{-1} \cdot\left[-G(q)+u+J(q)^{T} \cdot F_{e}\right]
$$

On this expression, the matrix $D(q)$ is invertible. It's a consequence of the leg mechanism design, specially chosen to avoid the singularities and allowing the leg to produce the required efforts. The state vector $q=\left[\begin{array}{lll}q_{1} & q_{2} & q_{3}\end{array}\right]^{T}$, denoting the joint variables, determines uniquely the foot position. This vector is obtained by integrating the Eq. (25).

\subsection{The Workspace of the Leg Mechanisms}

The workspace for a given legs configuration of the robot consists of all possible translations and rotations for the robot components (robot body and leg links). The physical constraints of each joint, as well the free space restrictions, are also considered for the workspace determination. We search the intersection of the so-called kinematic and static workspace to have the resultant workspace.

This approach is usually applied in geometry optimisation of the mechanism design, determination of the number of joints and selection of the active joints. It's also applied in the determination of forces and torques on the active joints, and computation of force distribution among supporting legs (Klein \& Kittivatcharapong, 1990; Zhang et al., 1996a; Zhang et al., 1996b).

There are two methods that can be used to analyse the leg kinematic workspace: the forward analysis, and the inverse analysis. Forward analysis determines the workspace using a function of space configuration $w=f(q)$, with $q=\left[\begin{array}{lll}q_{1} & q_{2} & q_{3}\end{array}\right]^{T}$, considering the physical limits for $q$. Inverse analysis determines the workspace through the inverse function, i.e. mapping the function $q=g(w)$ for a given mechanism position and orientation, and verifying if the configuration relative to $q$ is located inside the allowed space.

The kinematic workspace in this work is investigated by the inverse kinematics equations. Four constraints must be taken into account in the kinematic workspace analysis of the leg mechanism: the joints coordinates, the leg velocity limit, the leg acceleration limit, and the geometric interference of the leg.

Considering the performance of the present available actuators, and the development of geometric studies concerning the robot platform, we can say that the main constraints to the velocity and acceleration limits of the leg movements are the physical joints limits and its 
geometric interference (Zhang et al., 1996a). Therefore, the problem constraints can be expressed by:

$$
\theta_{\min } \leq \theta_{i j} \leq \theta_{\max }
$$

where $\theta_{i j}$ is the $j$-th joint of the $i$-th leg. Rewriting this equation using a vector of kinematics' constraints, we have:

$$
q_{\min } \leq q_{j} \leq q_{\max }
$$

where $q_{\min }=\left[\theta_{1 \min } \theta_{2 \min } \theta_{3 \min }\right]^{\mathrm{T}}$ and $q_{\max }=\left[\theta_{1 \max } \theta_{2 \max } \theta_{3 \max }\right]^{\mathrm{T}}$.

The variation of $\theta_{i j}$ angles for a specific $i$-th leg is function of the robot body position and orientation. The $i$-th foot position can be obtained by inverse analysis. Considering the feet positions, the position and the orientation of the robot body, we can compute $q_{=}=\left[\begin{array}{lll}q_{1} & q_{2} & q_{3}\end{array}\right]^{T}$. The difference vectors, $\Delta q_{\max }=q_{\max }-q_{i}$ and $\Delta q_{\min }=q_{\min }-q_{i}$, are used to determine when a boundary point is attained, i.e. if $\Delta q_{\max }$ or $\Delta q_{\min }$ is a null vector the leg configuration is on a workspace frontier. Scanning all the joint angles ranges, the total workspace of the leg mechanism is determined (Zhang et al. 1996a).

On the other hand, the study of the robot static workspace considers three types of constraints: the limits of force and torque on active joints, the maximum and minimum reaction forces on the soil-feet interface, and static friction coefficient of the feet-soil contact.

The expression of these constraints, considering the $j$-th joint of the $i$-ith robot leg, is given by:

$$
\begin{aligned}
& F_{\min } \leq F_{i j} \leq F_{\max } \\
& T_{\min } \leq T_{i j} \leq T_{\max } \\
& f_{\min } \leq f_{i z} \leq f_{\max } \\
& \mu_{t}=f_{x y} / f_{z}=\left(f_{x}^{2}+f_{y}^{2}\right)^{1 / 2} / f_{z} \leq \mu
\end{aligned}
$$

where $F_{i j}$ is the joint force, $T_{i j}$ is the joint torque, $f_{i z}$ is the reaction force on the soil-foot interface, and $\mu$ is the static friction coefficient. The ratio $\mu_{t}$ between the tangent and normal components of the reaction force on the soil-foot contact must respect the friction constraint, i.e. $\mu_{t} \leq \mu$ (Klein \& Kittivatcharapong, 1990; Martins-Filho \& Prajoux, 2000; Zhang et al., $1996 \mathrm{~b})$. For the analysis of the constraints listed above, we consider the static constraint vector defined by:

$$
\begin{aligned}
& c_{\max }=\left[\begin{array}{llll}
F_{\max }^{T} & T_{\max }^{T} & f_{\max }^{T} & \mu
\end{array}\right] \\
& c_{\min }=\left[\begin{array}{llll}
F_{\min }^{T} & T_{\min }^{T} & f_{\min }^{T} & \mu
\end{array}\right]
\end{aligned}
$$


Considering the feet positions, the position and the orientation of the robot body, we can compute $c=\left[\begin{array}{llll}F_{i j}{ }^{T} & T_{i j}{ }^{T} & f_{i j}{ }^{T} & \mu_{t}\end{array}\right]^{T}$. The difference vectors, $\Delta c_{\max }=c_{\max }-c_{i}$ and $\Delta c_{\min }=c_{\min }-c_{i}$, are used to determine when a boundary point is attained, i.e. if $\Delta c_{\max }$ or $\Delta c_{\min }$ is a null vector the leg configuration is on a workspace frontier. Scanning all the $c$ vector range, the total static workspace of the leg mechanism is determined (Zhang et al. 1996b).

Graphical representations of the kinematic and static workspaces, considering the adopted quadruped robot and leg mechanisms design, are shown in the Fig. 3 and Fig. 4, respectively.

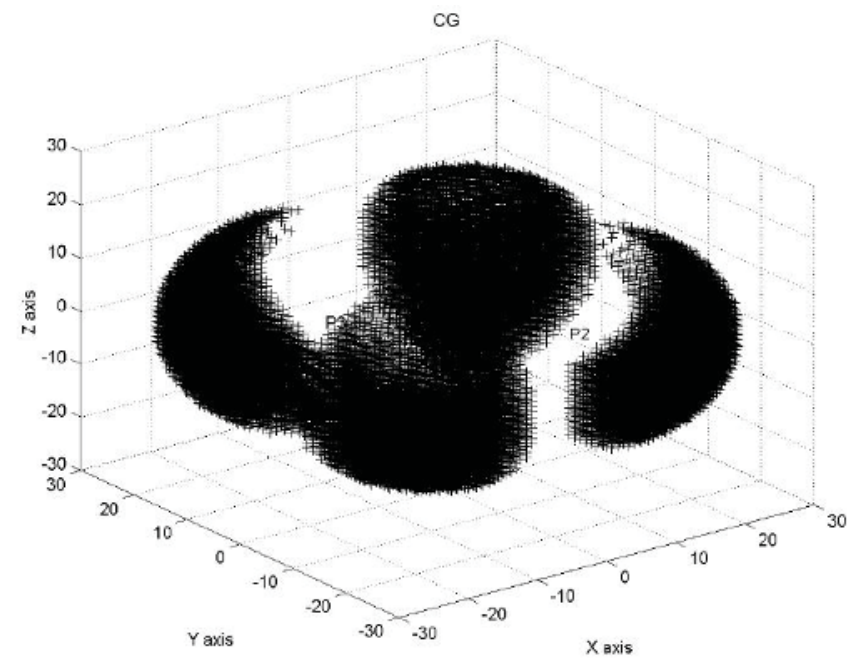

Fig. 3. The kinematic workspace of the studied quadruped robot

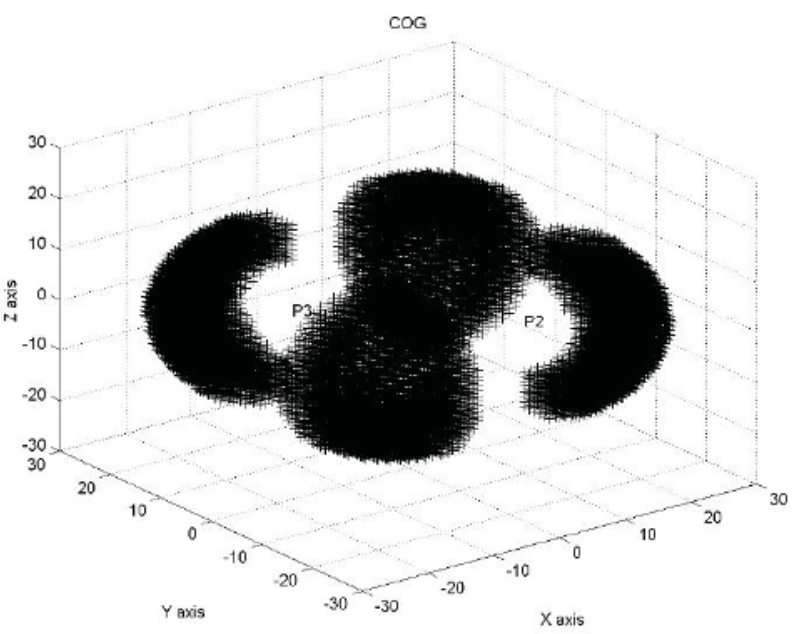

Fig. 4. The static workspace of the studied quadruped robot 


\section{Analysis of Matrices $C(q . d q / d t)$ and $G(q)$}

The position control problem of a system represented by the Lagrangian formulation can be solved through the application of a PD (proportional plus derivative) control approach. The PD control law is defined to determine the control torque to be produced on the active joints (Bucklaew et al., 1999). The expression of this proposed control is given by:

$$
u=K_{p} \cdot \tilde{q}+K_{d} \cdot \dot{\tilde{q}}
$$

where $K_{p}$ and $K_{d}$ are positive define matrices with constant gain values, and $\tilde{q}=\left(q_{d}-q\right)$ is the position error.

Applying this control law on the expression of $d^{2} q / d t^{2}$, the vector $d q / d t$ can be determined, and the vector $q$, the leg joints state, is available by integrating the differential equation. In this equation, the matrices $C(q, d q / d t)$ and $G(q)$ appear explicitly. Theses matrices must be computed at each new value of $q$ and $d q / d t$, and these quantities change continuously with the time. For a system with tree degrees-of-freedom or more, as the case of the robot considered in this work, the computation requires a considerable number of arithmetic operations and amount of time of controller processing. This system is supposed to be working on real-time, and this computation charge and time can represent a difficulty or trouble.

The elements of the matrix $C(q, d q / d t)$ are computed using the matrix $D(q)$. And this matrix $D(q)$ is a function of the links' masses, of the linear and angular velocities of these links, and it is also a function of the links' moments of inertia (taken on their own centres-of-mass). It is computed at each variation step of $q$. If the masses of the robot's links are small comparing to the body mass, that's the case of the quadruped robot considered in this work, the respective moments of inertia are also small. Analysing the velocities, the linear and the angular, we see that if the robot is moving relatively slow (that's the case of when the robot is performing a safe and stable gait), these velocities values are low. The contribution of another term, the Coriolis force, is dependent of the link velocity, consequently the relative significance of the matrix $C(q, d q / t)$ is low. The same conclusion can be taken of the analysis of the matrix $G(q)$, the gravitational contribution, that's a direct function of the links' masses.

\section{Numerical Simulations and Results}

The proposed model simplification was tested through numerical simulations. The motion of the robotic leg mechanism was simulated using two dynamical modelling, the complete one and the simplified one. For these simulations, a closed loop control scheme was defined applying the PD strategy to define the active joints torques to be produced by the servomotors. The control of the leg motion aims to track desired trajectories of the joint angles vector. The analysis of the results is based on the comparative performance of the two different models and on the verification of the tracking errors.

The simulation scenarios are defined in terms of leg manoeuvres involving the three active joints of the mechanism, taking into account the leg workspace limits. The values of the mechanism's physical parameters are defined based on a realistic future realization of a leg 
prototype. These parameters and the respective values considered on the numerical simulations are shown in the Tab. 1.

\begin{tabular}{|c|c|}
\hline extension of lifting and landing $(m)$ & $0.10 \pm 0.010$ \\
\hline displacements $(m)$ & $\begin{array}{c}\text { longitudinal: } 0.08 \pm 0.008 \\
\text { vertical: } 0.06 \pm 0.006 \\
\text { lateral: } 0.05 \pm 0.005\end{array}$ \\
\hline loads $(\mathrm{kg})$ & $\begin{array}{c}\text { vertical: } 4.5 \pm 0.45 \\
\text { horizontal: } 1.0 \pm 0.1 \\
\\
\text { lateral: } 0.5 \pm 0.05\end{array}$ \\
\hline geometrics dimensions $(\mathrm{m})$ & $\begin{array}{c}\text { first link: } 0.12 \pm 0.012 \\
\text { second link: } 0.08 \pm 0.008 \\
\text { link thickness: } 0.02 \pm 0.002\end{array}$ \\
\hline number of joints & 3 \\
\hline weight $(\mathrm{kg})$ & $2.0 \pm 0.2$ \\
\hline
\end{tabular}

Table 1. The considered values of the leg mechanism's parameters

The first manoeuvre concerns the first joint, commanding the corresponding servo from an initial configuration $q_{\text {init }}=\left[\begin{array}{lll}\theta_{1} & \theta_{2} & \theta_{3}\end{array}\right]^{T}=\left[\begin{array}{lll}0^{\circ} & 0^{\circ} & 90^{\circ}\end{array}\right]^{T}$ to a final configuration $q_{f i n}=\left[\begin{array}{lll}\theta_{1} & \theta_{2} & \theta_{3}\end{array}\right]^{T}=$ $\left[\begin{array}{lll}45^{\circ} & 0^{\circ} & 90^{\circ}\end{array}\right]^{T}$, and returning to the initial configuration. The results for the numerical simulation of the complete model and the simplified model can be seen in Figs. 5 and 6 , respectively.

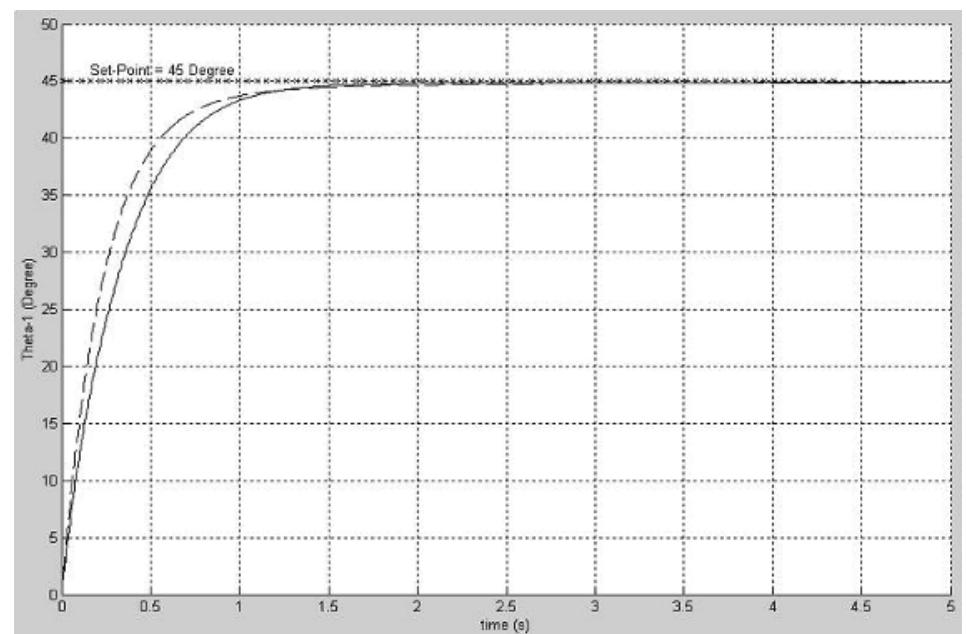

Fig. 5. Results of numerical simulation of the first step of the first manoeuvre (complete model "_- and simplified model "- - -")

In the second manoeuvre, the leg mechanism was commanded using the second joint. The servo departs from an initial configuration $q_{\text {init }}=\left[\begin{array}{lll}\theta_{1} & \theta_{2} & \theta_{3}\end{array}\right]^{T}=\left[\begin{array}{lll}90^{\circ} & 0^{\circ} & 50^{\circ}\end{array}\right]^{T}$ and it is commended to a final configuration $q_{f i n}=\left[\begin{array}{lll}\theta_{1} & \theta_{2} & \theta_{3}\end{array}\right]^{T}=\left[\begin{array}{lll}90^{\circ} & 30^{\circ} & 50^{\circ}\end{array}\right]^{T}$, and returning to the 
initial configuration. The results for the numerical simulation of the complete model and the simplified model can be seen in Figs. 7 and 8, respectively.

In the third and last manoeuvre, the leg mechanism was commanded using the third joint. The servo departs from an initial configuration $q_{\text {init }}=\left[\begin{array}{lll}\theta_{1} & \theta_{2} & \theta_{3}\end{array}\right]^{T}=\left[\begin{array}{lll}90^{\circ} & 0^{\circ} & 90^{\circ}\end{array}\right]^{T}$ and it is commended to a final configuration $q_{f i n}=\left[\begin{array}{lll}\theta_{1} & \theta_{2} & \theta_{3}\end{array}\right]^{T}=\left[\begin{array}{lll}90^{\circ} & 30^{\circ} & 45^{\circ}\end{array}\right]^{T}$, and returning to the initial configuration. The results for the numerical simulation of the complete model and the simplified model can be seen in Figs. 9 and 10, respectively.

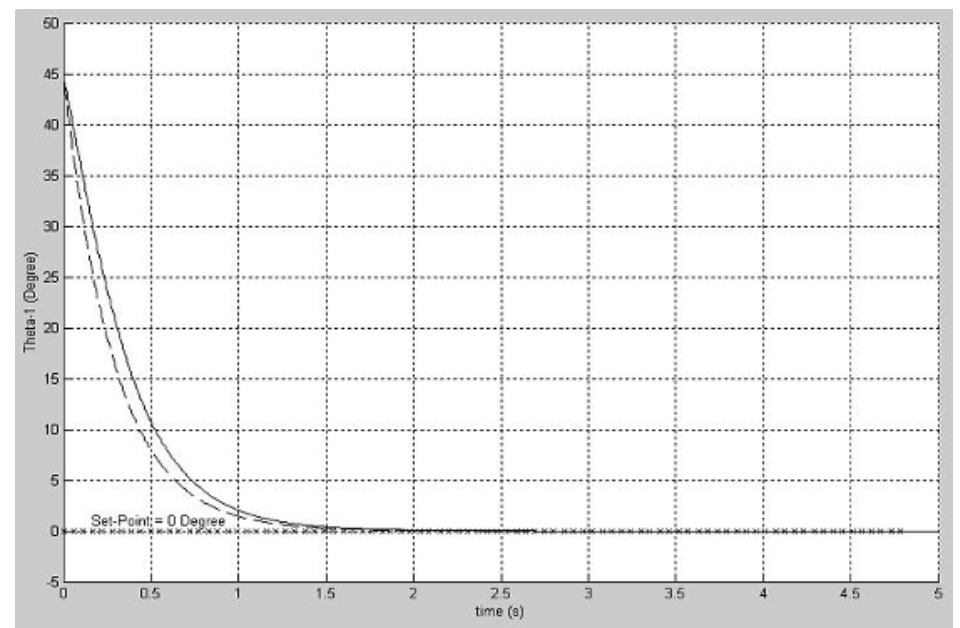

Fig. 6. Results of numerical simulation of the second step of the first manoeuvre (complete model "_-" and simplified model "- - -")

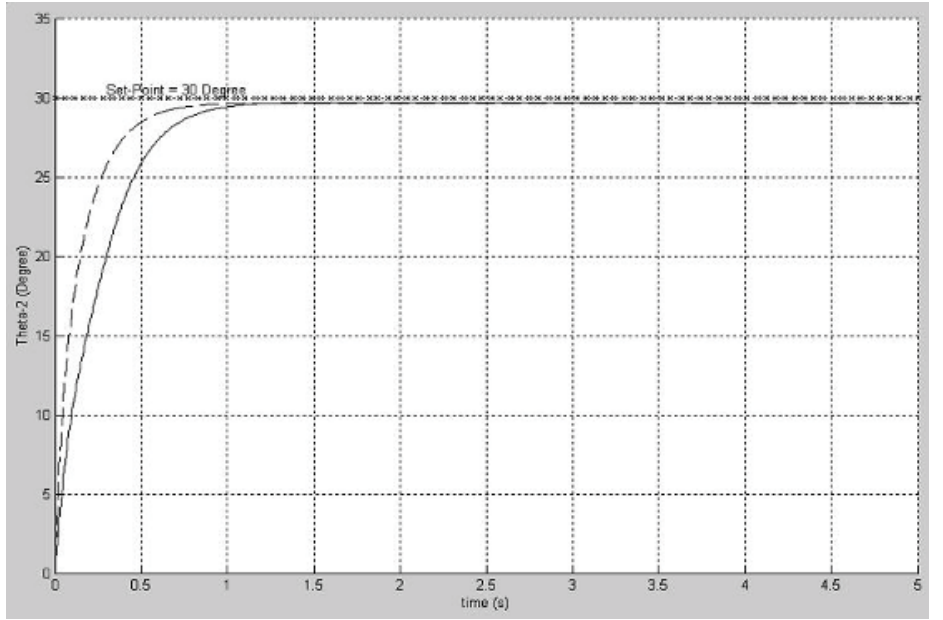

Fig. 7. Results of numerical simulation of the first step of the second manoeuvre (complete model "_- " and simplified model "- - -") 


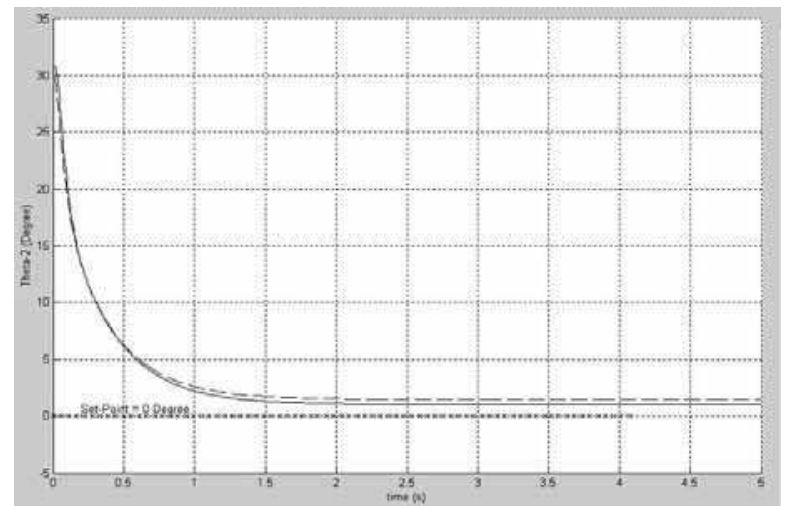

Fig. 8. Results of numerical simulation of the second step of the second manoeuvre (complete model "__" and simplified model " - - -")



Fig. 9. Results of numerical simulation of the first step of the third manoeuvre (complete model "_- and simplified model "- - -")

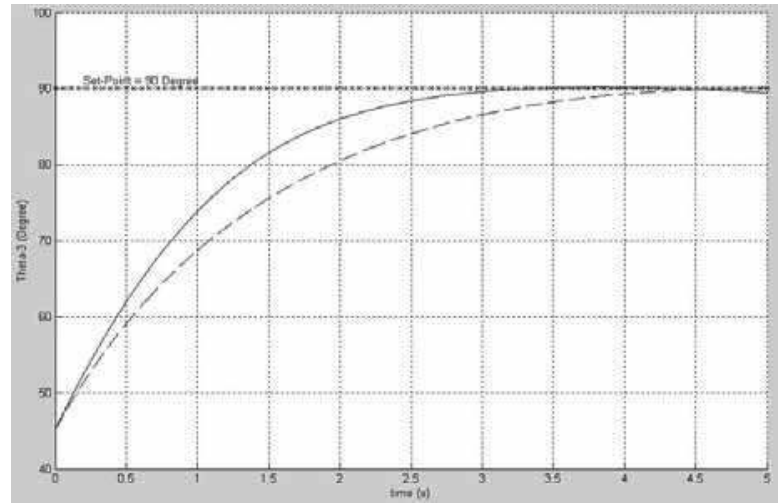

Fig. 10. Results of numerical simulation of the second step of the third manoeuvre (complete model "—" and simplified model "- - -") 
Considering the obtained results of numerical simulations (see Figs. 5, 6, 7, 8, 9 and 10), we can verify that the dynamical responses are approximately similar for the two models of the robot dynamics, with and without the matrices $C(q, d q / d t)$ and $G(q)$. The difference between the two models are limited to around 2 degrees on the steady state behaviour of the amplitude, and a delay around 10 degrees on the transitory phase.

As an immediate consequence, the number of calculation operations for the determination of the value of robot's joints state, using the system accelerations, is significantly reduced. In terms of computation time, the estimation of gain is around $9 \%$ of reduction.

\section{Conclusion}

In this work, a dynamical model simplification was implemented and analysed considering a small quadruped mobile robot, equipped with pantographic leg mechanisms. This specific leg design and characteristics were considered, as well the context of realistic possible physical realization (dimensions, masses, inertias) of a laboratory prototype. A simplification was proposed aiming the reduction of computational efforts for the evaluation of the matrices $C(q, d q / d t)$ and $G(q)$, corresponding respectively to the centrifugal and Coriolis forces, and to the gravitational dynamical contributions.

The resulting advantages of the presented proposition were verified when comparing the numerical simulations of the leg mechanism motions taking the complete and simplified models. The obtained results of dynamical performance for both cases are very similar, i.e. the difference is around 1 degree on steady state, and a delay bellow 2 degrees on the transitory phase. On the other hand, the computation time reduction using the simplified model is quite expressive (around 9\%). These two facts confirm the validity of using the proposed simplified model, it can be useful on experimental applications of locomotion control approaches based on the dynamical model of the walking robot. For instance, this time reduction can be advantageous for pre-computed torque and feedforward control approaches. Considering the critical requirements of the real-time control of a complex system as a legged mobile robot.

The future works include the construction of the leg mechanism and robot prototypes, the application of the simplifications proposed here on this experimental legged robot, and the analysis of this system performance using diverse force control approaches.

\section{References}

Asada, H. and J. J. E. Slotine (1986). Robot Analysis and Control. Ed. Wiley-Interscience Publications, New York, USA.

Baroni, P., G. Guida, S. Mussi and A. Venturi (1995). A distributed architecture for control of autonomous mobile robots. Proceedings of IEEE International Conference on Robotics and Automation. Nagoia, Japan.

Bucklaew, T. P. and C.-S. Liu (1999). A new nonlinear gain structure for PD-type controllers in robotic applications. Journal of Robotic Systems, Vol. 16, No. 11, pp. 627-649.

Craig, J. J. (1986). Introduction to Robotics Mechanics and Control. Ed. Addison Wesley Publishing Company, Stanford University, USA. 
Cunha, A.C., C. C. Bier, D. Martins and F. Passold (1999). Metodologia seqüencial para simulação numérica de técnicas de controle para robôs manipuladores (in portuguese). Proceedings of Iberian Latin-American Congress on Computational Methods in Engineering. EPUSP, São Paulo, Brazil.

Fu, K. S.; R. C. Gonzalez and C. S. G. Lee (1987). Robotics: control, sensing, vision and intelligence. Ed. McGraw-Hill Book Company, New York, USA.

Hirose, S., K. Yoneda, R. Furuya and T. Takagi (1989). Dynamic and static fusion control of quadruped walking vehicle. Proceedings of IEEE International Conference on Intelligent Robots and Systems. Tsukuda, Japan.

Klein, C.A. and S. Kittivatcharapong (1990). Optimal force distribution for the legs of a walking machine with friction cone constraints. IEEE Transactions on Robotics and Automation. Vol. 6, No. 1, pp. 73-85.

Martins-Filho, L. S. and R. Prajoux (2000). Locomotion control of a four-legged embedding real-time reasoning in the force distribution. Robotics and Autonomous Systems, Vol. 32, No. 4, pp. 219-235.

Medeiros, A.D., R. Chatila and S. Fleury (1996). Specification and validation of a control architecture for autonomous mobile robots. Proceedings of IEEE International Conference on Intelligent Robots and Systems. Osaka, Japan.

Schneider, A. and U. Schmucker (2001). Forced legged platform KATHARINA for service operations, Proceedings of the International Conference on Climbing and Walking Robots, Karlsruhe, Germany.

Spong, M. W. and M. Vidyasagar (1989). Robot Dynamics and Control. Ed. John Wiley and Sons, New York, USA.

Tanie, K. (2001). New trends of walking robotics research and its application possibilities, Proceedings of the International Conference on Climbing and Walking Robots, Karlsruhe, Germany.

Zhang, D.J. Sanger and D. Howard (1996), Workspaces of a walking machine and their graphical representation. Part I : Kinematic Workspaces, Robotica. Vol. 14, No. 1, pp. 71-79.

Zhang, S.J., D.J. Sanger and D. Howard (1996), Workspaces of a walking machine and their graphical representation. Part II : Static Workspaces, Robotica. Vol. 14, No. 2, pp. 219-226. 


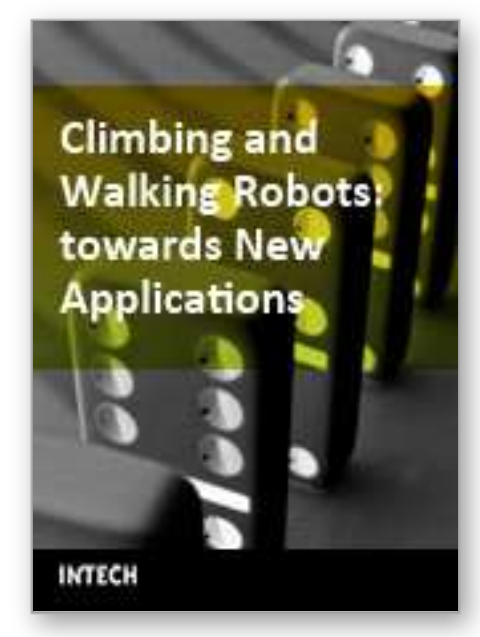

\author{
Climbing and Walking Robots: towards New Applications \\ Edited by Houxiang Zhang
}

ISBN 978-3-902613-16-5

Hard cover, 546 pages

Publisher I-Tech Education and Publishing

Published online 01, October, 2007

Published in print edition October, 2007

With the advancement of technology, new exciting approaches enable us to render mobile robotic systems more versatile, robust and cost-efficient. Some researchers combine climbing and walking techniques with a modular approach, a reconfigurable approach, or a swarm approach to realize novel prototypes as flexible mobile robotic platforms featuring all necessary locomotion capabilities. The purpose of this book is to provide an overview of the latest wide-range achievements in climbing and walking robotic technology to researchers, scientists, and engineers throughout the world. Different aspects including control simulation, locomotion realization, methodology, and system integration are presented from the scientific and from the technical point of view. This book consists of two main parts, one dealing with walking robots, the second with climbing robots. The content is also grouped by theoretical research and applicative realization. Every chapter offers a considerable amount of interesting and useful information.

\title{
How to reference
}

In order to correctly reference this scholarly work, feel free to copy and paste the following:

Jose L. Silvino, Peterson Resende, Luiz S. Martins-Filho and Tarcisio A. Pizziolo (2007). Simplified Modelling of Legs Dynamics on Quadruped Robots' Force Control Approach, Climbing and Walking Robots: towards New Applications, Houxiang Zhang (Ed.), ISBN: 978-3-902613-16-5, InTech, Available from: http://www.intechopen.com/books/climbing_and_walking_robots_towards_new_applications/simplified_modelli ng_of_legs_dynamics_on_quadruped_robot_s_force_control_approach

\section{INTECH}

open science | open minds

\author{
InTech Europe \\ University Campus STeP Ri \\ Slavka Krautzeka 83/A \\ 51000 Rijeka, Croatia \\ Phone: +385 (51) 770447 \\ Fax: +385 (51) 686166 \\ www.intechopen.com
}

\author{
InTech China \\ Unit 405, Office Block, Hotel Equatorial Shanghai \\ No.65, Yan An Road (West), Shanghai, 200040, China \\ 中国上海市延安西路65号上海国际贵都大饭店办公楼 405 单元 \\ Phone: +86-21-62489820 \\ Fax: +86-21-62489821
}


(C) 2007 The Author(s). Licensee IntechOpen. This chapter is distributed under the terms of the Creative Commons Attribution-NonCommercial-ShareAlike-3.0 License, which permits use, distribution and reproduction for non-commercial purposes, provided the original is properly cited and derivative works building on this content are distributed under the same license. 